University of Wollongong

Research Online

SMART Infrastructure Facility - Papers

Faculty of Engineering and Information

Sciences

$1-1-2018$

\title{
An Ontology Based Context-Aware Architecture for Smart Campus Applications
}

\author{
Nicolas R. Verstaevel \\ University of Wollongong, nicolasv@uow.edu.au \\ Guillaume Garzone \\ University of Toulouse \\ Thierry Monteil \\ University of Toulouse \\ Nawal Guermouche \\ University of Toulouse \\ Johan Barthelemy \\ University of Wollongong, johan@uow.edu.au
}

See next page for additional authors

Follow this and additional works at: https://ro.uow.edu.au/smartpapers

Part of the Engineering Commons, and the Physical Sciences and Mathematics Commons

Research Online is the open access institutional repository for the University of Wollongong. For further information contact the UOW Library: research-pubs@uow.edu.au 


\title{
An Ontology Based Context-Aware Architecture for Smart Campus Applications
}

\begin{abstract}
With an estimated number of more than 15 billion, objects, the management of architectures for the Internet of Things is a veritable challenge. The inherent mobility (in terms of devices and users) of the loT means that the architecture has to be resilient to appearance and disappearance of devices. In this paper, we address the problem of autonomic management of loT architecture by the means of ontologies. The problem we address is that given a dynamic system which is built upon a multitude of entities abstracted as services and characterized by their inputs and outputs, evolving targets that aim to provide services in terms of data or in terms of control, our goal is to enable autonomic management of these kinds of systems to cope with changes and evolutions so that the specified targets are fulfilled throughout the execution according to the specifics and dynamic needs of the system's users. We propose an innovative architecture that relies on ontologies to enable context-aware application to self-compose on demand. This architecture is being deployed to two smart campuses in two universities from Toulouse, France and Wollongong, Australia.
\end{abstract}

\section{Keywords}

ontology, architecture, campus, context-aware, applications, smart

Disciplines

Engineering | Physical Sciences and Mathematics

\section{Publication Details}

Verstaevel, N., Garzone, G., Monteil, T., Guermouche, N., Barthelemy, J. \& Perez, P. (2018). An Ontology Based Context-Aware Architecture for Smart Campus Applications. 2018 IEEE Intl Conf on Parallel \& Distributed Processing with Applications, Ubiquitous Computing \& Communications, Big Data \& Cloud Computing, Social Computing \& Networking, Sustainable Computing \& Communications (pp. 1056-1063). United States: IEEE.

\section{Authors}

Nicolas R. Verstaevel, Guillaume Garzone, Thierry Monteil, Nawal Guermouche, Johan Barthelemy, and Pascal Perez 


\title{
An ontology based context-aware architecture for smart campus applications
}

\author{
Nicolas Verstaevel $^{1}$, Guillaume Garzone ${ }^{2}$, Thierry Monteil ${ }^{2}$, Nawal Guermouche ${ }^{2}$, Johan Barthelemy ${ }^{1}$, Pascal Perez ${ }^{1}$ \\ ${ }^{1}$ SMART Infrastructure Facility, University of Wollongong, Wollongong, Australia \\ ${ }^{2}$ LAAS-CNRS, Université de Toulouse, INSA Toulouse, France
}

\begin{abstract}
With an estimated number of more than 15 billion objects, the management of architectures for the Internet of Things is a veritable challenge. The inherent mobility (in terms of devices and users) of the IoT means that the architecture has to be resilient to appearance and disappearance of devices. In this paper, we address the problem of autonomic management of IoT architecture by the means of ontologies. The problem we address is that given a dynamic system which is built upon a multitude of entities abstracted as services and characterized by their inputs and outputs, evolving targets that aim to provide services in terms of data or in terms of control, our goal is to enable autonomic management of these kinds of systems to cope with changes and evolutions so that the specified targets are fulfilled throughout the execution according to the specifics and dynamic needs of the system's users. We propose an innovative architecture that relies on ontologies to enable context-aware application to selfcompose on demand. This architecture is being deployed to two smart campuses in two universities from Toulouse, France and Wollongong, Australia.
\end{abstract}

Index Terms-smart campus, dynamic service management, IoT, semantic, graph grammar

\section{INTRODUCTION}

The Internet of Things (IoT) has been defined by ITU [1] as a global infrastructure for the information society, enabling advanced services by interconnecting (physical and virtual) things based on existing and evolving interoperable information and communication technologies. This simple definition shows that several networks will interact, that there will be both physical entities (such as a presence sensor or a controllable lamp) but also virtual entities (for example, all the lamps of a building) and finally, the last important point is that it will be necessary to be able to communicate and access the services proposed by all objects. The domains covered by IoT are very large: smart factory, smart grid, smart cities, etc. This article will focus on smart campus infrastructure and scenarios. Smart campuses are a great environment for experiments. They are in fact small cities inside the city. They have the problem of energy saving, mobility, optimization of space and building occupation, high level of service for students, etc. They have built smart buildings and are able to deploy real experiments for their users. The students are also good consumers of existing and new technology.

It is estimated that the range of objects will grow from 15 to 49 billion. Even if for a specific usage the number of objects accessible is not so high, there are many challenges to solve due to the IoT systems: the number of devices, the interoperability between technologies, the data collected or the dynamic of architecture. Other challenges come from users due to the mobility, the diversity of usage or the necessary respect of privacy. Much work has been done to create valid architectures and standardized IoT architectures which take care of interoperability problem. The goal is now to use those architectures to produce applications for users. In this paper, we focus on the problem of creating an application that uses a highly dynamic IoT service platform in a mobility context applied to the smart campus of the University of Wollongong. The problem is to describe and discover dynamically the IoT services platforms and to use the several services offered by the devices to compose on-the-fly new applications. The paper is structured as follows: in section II the motivating example in the smart campus context is described. Then in section III the standardized IoT architecture and dynamic service management infrastructure are explained. The section IV is dedicated to the extended ontology proposed to capture the relationship between users' needs and services offered in a specific context. Experimentation description is done in section V. Before concluding, related work are given in section VI.

\section{Motivating EXAMPLe}

In this paper, we consider a smart campus scenario. Smart Campus' have proven to be good places for in vivo experimentation [12] [13]. Indeed, a University Campus is generally spread on several hectares and populated with a lot of buildings, lawns, and transport infrastructures. Each building may have multiple purposes, teaching, research, food hall and so on. Furthermore, each building has been designed separately with its own infrastructure. They are frequented by several types of end-users with different needs and habits. Students, faculty members, university staff, service providers, visitors and so on meet throughout the day leading to a dynamic flow of human activities. As a city, the campus lives on energy (water, electricity) and produces waste that must be evacuated. So, at a smaller scale, a campus experiences the challenges of nowadays cities.

In this scenario, different entities exchange services. A service can be any information or data that is made available (i.e., data form sensors, software services, cloud platform) or 
the control over a resource (i.e. access to a screen, control of light levels, control over windows). Each building has its own set of services distributed among the different rooms. Some of those services are linked with a specific device (for example, a screen or a light), whereas others are linked to a room or to the building itself. Different users (students, teachers, technicians, administration) are moving from building to buildings as part of their everyday activities. Each user has a specific (and sometime unique) interest for different kinds of services, depending on their interests, current activity and location. Furthermore, accessibility to services may be different depending on the type of user and the time of the day (for example, access to some building resources by the students may be forbidden during nights), or varying to building management strategies.

In this scenario, two types of entities can be considered:

- Interaction enablers: entities that create and provide services to building users.

- Interaction consumers: entities that are interested in accessing to specific topics according to their interaction interests. For instance, a teacher might be interested in access to the devices in a teaching room, whereas students might only consider information about building access to bathrooms or drinking fountains.

Therefore, different kinds of services are produced and provided by interaction enablers. Interaction consumers express their interests for certain types of services, and according to these interests, can be connected with different set of services. Depending of the type of user and its context (this notion is discussed in section IV-B), a user can be granted access to a service, and thus, a specific composition between the service and the user occurs.

Users can dynamically express different kinds of interests to a multitude of services that can be composed dynamically by different providers (connected devices, software components, etc). A teacher may be interested in having access to diffusion screen, a building administrator to metrics about the buildings energy performance, and a student to the location of facilities within the campus. Each user has specific and dynamic needs. Furthermore, as some interaction enablers can be mobile (for example, IoT devices or smartphones), and the different users are moving within the campus, switching from one building to the other, the interaction enablers and interaction consumers can appear and disappear at runtime.

In such a context, it is difficult to specify a priori all the interactions that may occur in such a dynamic system, giving to the system the ability to self-adapt to changes and evolutions while guaranteeing the satisfaction of users is mandatory. Such self-adaptation process must take into account the context of each entity in order to enable composition on-the-fly.

Thus, the problem can be described as follow:

Given a dynamic system which is built upon a multitude of entities abstracted as services and characterized by their inputs and outputs, evolving targets that aim to provide services in terms of data or in terms of control, our goal is to enable autonomic management of this kind of systems to cope with changes and evolutions so that the specified targets are fulfilled throughout the execution according to the specifics and dynamic needs of the systems users.

In the next section, we propose an architecture addressing this need.

\section{SySTEM ARCHITECTURE}

\section{A. IoT Infrastructure}

The implementation of an IoT architecture is often based on an existing one that is being enriched. It is rare to have a monolithic architecture in terms of technology, and even if it may be true at the moment of deployment, the evolutions of the system and the expectations don't remain true in time. The interoperability problem and the access to the service offered becomes a key point for an IoT architecture to be viable over time.

Creating an architecture based on standards is a key point. The oneM2M standard ${ }^{1}$ is made up of various standardization organizations around the world. oneM2M offers a fairly generic and horizontal vision of connected systems. It will position itself transversely, regardless of the field of application. The idea is that oneM2M creates a homogeneous vision of the system. This is done through a layer of services accessible through a standardized interface. The different underlying protocols are thus abstracted and included in a homogenized vision by using a specific service called Interworking Proxy Entity (IPE). The interoperability mechanism is completed by the use of semantic discovery. A oneM2M architecture is modeled by different entities (figure 1). At the application level, the AE for application entities will enable representation of the different applications connected to the IoT system whether they are high-level applications, users or even the objects themselves. Then, the service layer provided by the different systems is represented by Common Services Entities (CSEs). These entities will enable applications to register in their system and will provide the various services related to the standard. Finally, the lowest layer, linked to the physical transport of data on the communication networks will be articulated with the layers of services that will abstract it. The CSEs provide service as registration, communication, security, persistence, data access, etc.

Practically, oneM2M architecture is based on sensors and controllable connected objects (Application Service Node ASN for powerful object or Application Dedicated Node ADN). These objects are connected to a gateway (Middle Node - MN) that will ensure the ascent and descent of information from the server (Infrastructure Node IN). Users applications can connect to the server or to a gateway using the oneM2M API based on REST.

The Eclipse OM2M software ${ }^{2}$ is an open source software initially developed at LAAS-CNRS that implements oneM2M

\footnotetext{
${ }^{1}$ http://www.onem2m.org

${ }^{2}$ http://www.eclipse.org/om2m
} 


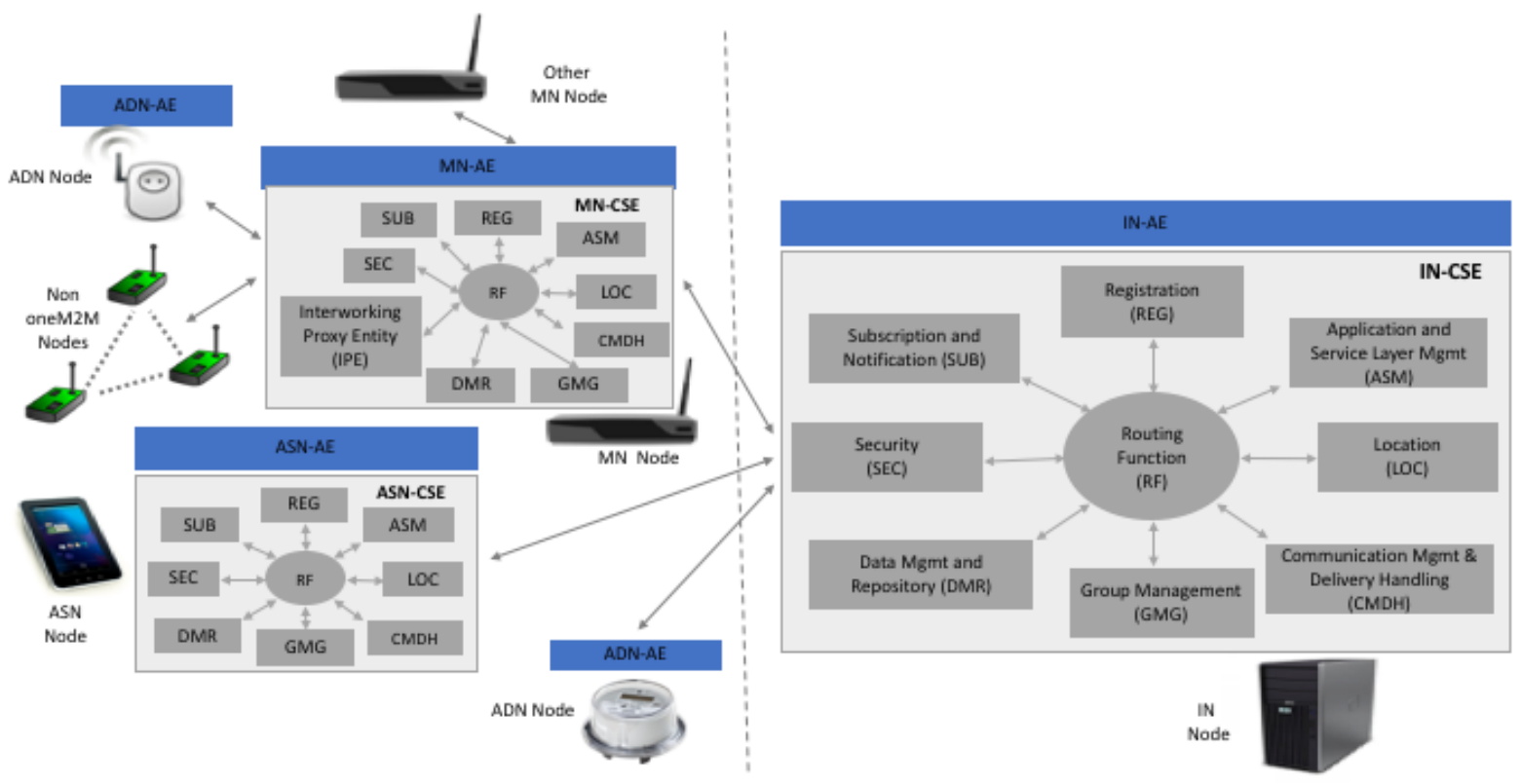

Fig. 1. oneM2M architecture

standard. It is used throughout the world both in academic and industrial sectors.

\section{B. Service management infrastructure}

The autonomic service management is based on MAPE-K loop developed in [4]. In [2] several models are used in the Knowledge Base $(K B)$ to interact with the IoT infrastructure deploy with OM2M. The Monitor and the Analyser use the ontology plus semantic reasoning and the Planner and the Executor use graph grammar rewriting rules to create actions to perform on IoT systems (figure 2). The main goal is to manage content and dynamic broadcast in a smart city and buses between citizens. Events are received from deployed IoT systems. Those events create instances inside the ontology. Then the Analyser infers knowledge using SWRL rules and a semantic reasoner. Based on the graph defined by this instance of the ontology, the Planer applies graph rewriting rules to obtain a new graph of possible connections between the needs of users and available services on the IoT architecture. Then, the Executor creates all connections using the REST architecture of OM2M.

\section{KNOWLEDGE AND REASONING MODELS}

One of the objectives of this paper is to generalize the ontology used in [2] for contents and dynamic broadcast to any interaction between an entity (human, building, vehicle, etc.) and an IoT system.

\section{A. Ontology model}

The proposed ontology extends the IoT-O ontology [3]. This ontology gathers several recognized ontologies to describe the
IoT system in terms of architecture, devices (sensors and activators), life cycle, energy and services offered. The extension proposed on figure 3 makes the connection between an entity (called interaction consumer) that expresses interaction interest with an IoT system. The IoT system through the interaction enabler allows to call service on devices. This interaction is controlled by the notion of interaction context defined in subsection IV-B also has the capability to share interaction between different interaction consumers, and devices.

\section{B. Interacting according to the Context}

In our scenario, interaction consumers seek to interact with interaction enablers in order to use on-the-fly a set of services. This composition is managed by the ontology and made possible if the two entities share similar interaction interest and if the interaction is allowed by the interaction

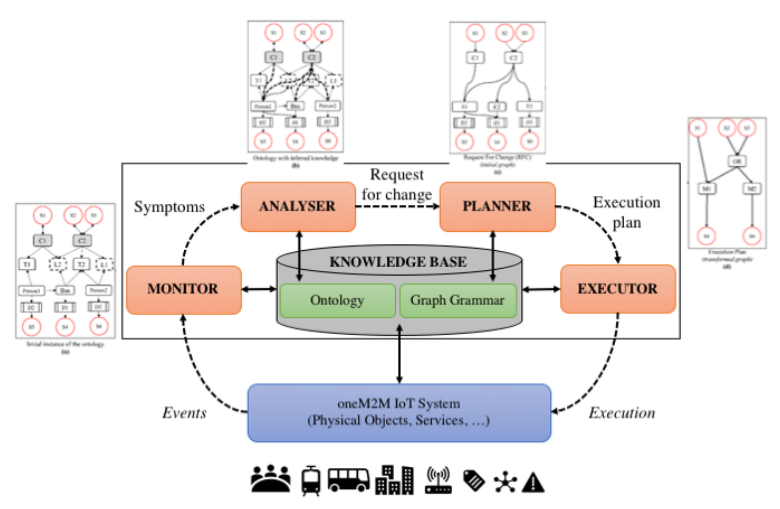

Fig. 2. Autonomic management for dynamic service 


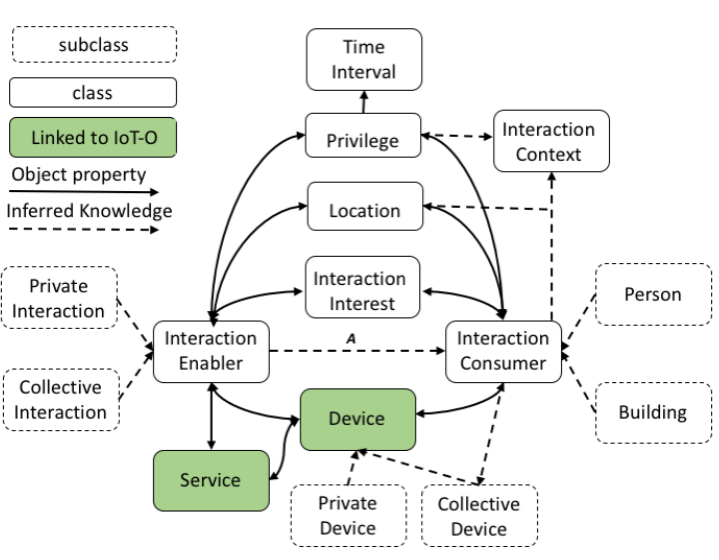

Fig. 3. The ontology based model for interaction between Users and the Smart campus building

context. The role of the interaction context is to ensure that the connection between the interaction consumers and the interaction enablers is allowed not only in terms of privileges but also in terms of location. As the term context is used in many scientific domains, there are several proposals for a definition of what context means [10]. In the field of problem solving, Brezillon defines the context as what constrains a step of a problem solving without intervening in it explicitly [7]. In the field of multi-agent systems, Guivarch et al. defines a context as all information which is external to the activity of an agent and affects its activity. It describes the environment as the agent sees it [6]. In this paper, we took the definition of Abowd et al. that defines context as any information that can be used to characterize the situation of an entity. An entity is a person, place, or object that is considered relevant to the interaction between a user and an application, including the user and applications themselves [9].

The term context awareness, also called sentient, was first introduced by Schilit and Theimer [8] in 1994. A later definition is provided by Abowd et al. as follows: A system is context-aware if it uses context to provide relevant information and/or services to the user, where relevancy depends on the users task [9].

Due to their dynamic nature and their heterogeneity, understanding sensor data is one of the main challenges that the IoT is facing. Perera et al. have identified context-awareness as an important topic in the IoT research field after evaluating a subset of 50 projects [11].

While the notion of context may include a variety of information to describe the environment of an entity, we propose in this article to add to the classical privilege information, information regarding connectivity as an element of context to describe the environment of an interaction consumer and to match this context with the environment of an interaction enabler in order to compose new services on-the-fly.

This idea is motivated by the dynamic nature of IoT systems in which mobile IoT devices and users evolve in interaction with various networks such as WiFi, Bluetooth Low Energy

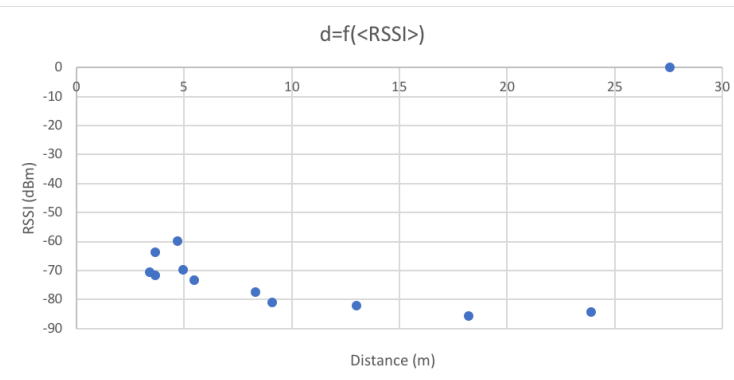

Fig. 4. Evolution of RSSI for BLE depending on the distance between the two devices

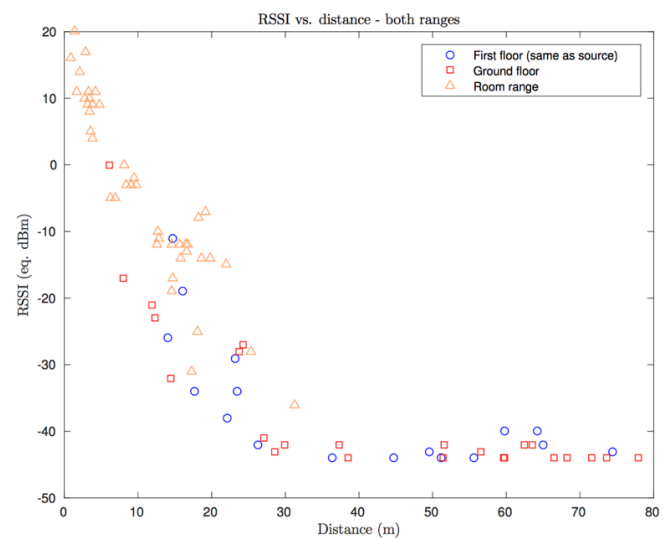

Fig. 5. Evolution of RSSI for LoRa depending on the distance between devices and gateway

(BLE) or LoRA. As those entities move, the type of available networks and the strength of signal is also evolving, and thus describing the environment of an entity. Figures 4 and 5 show results of an experiment in which we evaluate the strength of a signal according to the distance for two types of networks: BLE (Figure 4) and LoRA (Figure 5). Those graphs show that there is a relationship between the distance and the strength of a signal. However, each network has its own distance range allowing a different location precision (within $5 \mathrm{~m}$ to $15 \mathrm{~m}$ for BLE, and from $0 \mathrm{~m}$ to $60 \mathrm{~m}$ for LoRA).

Each interaction consumer and interaction consumer will describe its environment by frequently listing all the networks available and the associated signal strength. By doing so, each entity describes the context in which it is. The interaction context role is then to map those different contexts and to allow the connection between two entities if there is a context similarity.

\section{Reasoning and service management Processes}

New instances and new relations between the different instances of systems represented through the ontologies are created depending on events received by the monitoring component of the autonomic loop. Some relations are created through inference. This mechanism is based on SWRL rules [5] and semantic reasoning. For example, the SWRL rule on listing 1 creates the relation A depicted in figure 3 . The rule states that a consumer has expressed an interaction interest, the 
context is valid in terms of location and privilege and there is a service that enables this interaction.

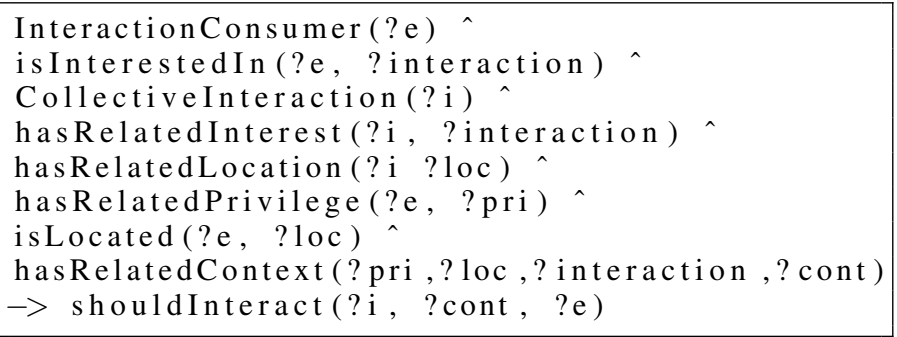

Listing 1. An example of a SWRL rule of the ontology

\section{EXPERIMENTATION}

\section{A. International IoT architecture}

Two different platforms has been deployed to create two smart-campus infrastructure (figure 6). The first one at the University of Wollongong (UOW) and the second at the LAAS-CNRS from University of Toulouse. Those two different infrastructures can cooperate and exchange information based on the same use of a standardized service layer based on oneM2M standard.

1) UOW smart campus: UOW has deployed a LoRa network at a regional scale based on the international open network The Things Network ${ }^{3}$. 8 outdoor gateways have been deployed around the city of Wollongong allowing a full coverage of the main Campus. In addition, smaller indoor gateways had been added to various buildings to increase geolocation capabilities. In the building 6 called SMART Infrastructure Facility, 125 LoRa sensors have been installed to monitor the environmental status of the rooms. Each device is connected through a LoRa network and has 5 sensors included (temperature, humidity, pressure, presence, battery). Other connected devices are also available to activate lamps, manage displays using several technologies to communicate like WIFI or Bluetooth Low Energy. Thanks to the oneM2M standard and the concept of Interworking Proxy Entity, services offered by this equipments are available through different oneM2M gateways (middle node $\mathrm{MN}_{-} *$ ). The architecture is completed with a cloud access using an infrastructure node of oneM2M called UOW_Smart_Campus. This allows a remote access using the Internet.

Specific Access Control Policies (ACP) to manage the different types of users, including Students, teachers, technical agents and international partners. ACP are used to create the concepts of privilege and time intervals described in the ontology (figure 3).

2) LAAS-CNRS smart campus: LAAS-CNRS has deployed a oneM2M architecture also based on the open source Eclipse OM2M middleware. A smart building called ADREAM building has been instrumented with several devices based on different technologies: EnOcean, Philips HUE and Zwave. A

\footnotetext{
${ }^{3}$ https://www.thethingsnetwork.org
}

specific oneM2M gateway is able to communicate with a robot based on the publish/subscribe protocol of ROS operating system. One of the main goals is to develop interaction between robot, human and environment ${ }^{4}$. The service layer of devices is accessible by the Internet thanks to the infrastructure node of oneM2M architecture.

\section{B. First scenario: Synthetic view of buildings}

In this first scenario, the objective is to give access to a technical agent to a synthetic view of the smart campus. For example, a technical agent would like to extract minimum temperature values collected inside all the campus buildings. First we create a virtual resource in one oneM2M infrastructure that will represent this value. This resource has an attribute of type semantic Descriptor that stores an instance of subset ontology described in figure 3 . This instance represents the interaction interest in terms of minimum, temperature value and building. This resource is also connected with the autonomic management system on figure 2 . The level of privilege of the technical agent allows him to get access to all resources that collect temperature information in both sites. The SWRL rules makes links between all those sensors located inside a building and also the service able to calculate a minimum between a set of values. Then the planner uses rewritten rules to make the connection between values represented by the selected resources and the minimum service. The value is then returned to the users using a node-RED flows ${ }^{5}$ made with IDE OM2M nodes (figure 7). It has to be noted that while sensors may be using different types of networks, the request is completely agnostic of any sensor protocol and only use the semantics associated to a value.

\section{Second scenario: Smart Teaching}

In this second scenario, the objective is to enable interaction between a teacher and their students during a lecture. A teacher wants to conduct real-time multiple choice quiz during the lecture in order to assess the level of understanding of the students. In this scenario, the teacher and the students act both as an interaction provider and an interaction consumer: the teacher provides questions and consumes answers, and the students consume questions and provide answers.

A virtual resource is created in a oneM2M infrastructure to model by the teacher. In this resource, three containers are created to store the questions, the answers and the description of the teacher context. This resource has an attribute of type semantic Descriptor that stores an instance of subset ontology described in figure 3 . This instance represents the interaction interests in terms of multiple choices questions, lecture subject, and teacher. The level of privilege of the teacher allows them to publish questions, consume answers and update its context.

Whenever a student launches the smart teaching application, the context of a student is extracted and the interaction context seeks for a valid teacher application to connect the student to. If the interaction context manages to find a matching resource,

\footnotetext{
${ }^{4}$ https://homepages.laas.fr/monteil/drupal/content/HumanRobotEnvironment
} ${ }^{5} \mathrm{https} / / /$ homepages.laas.fr/monteil/drupal/content/node-red-ideom $2 \mathrm{~m}$ 


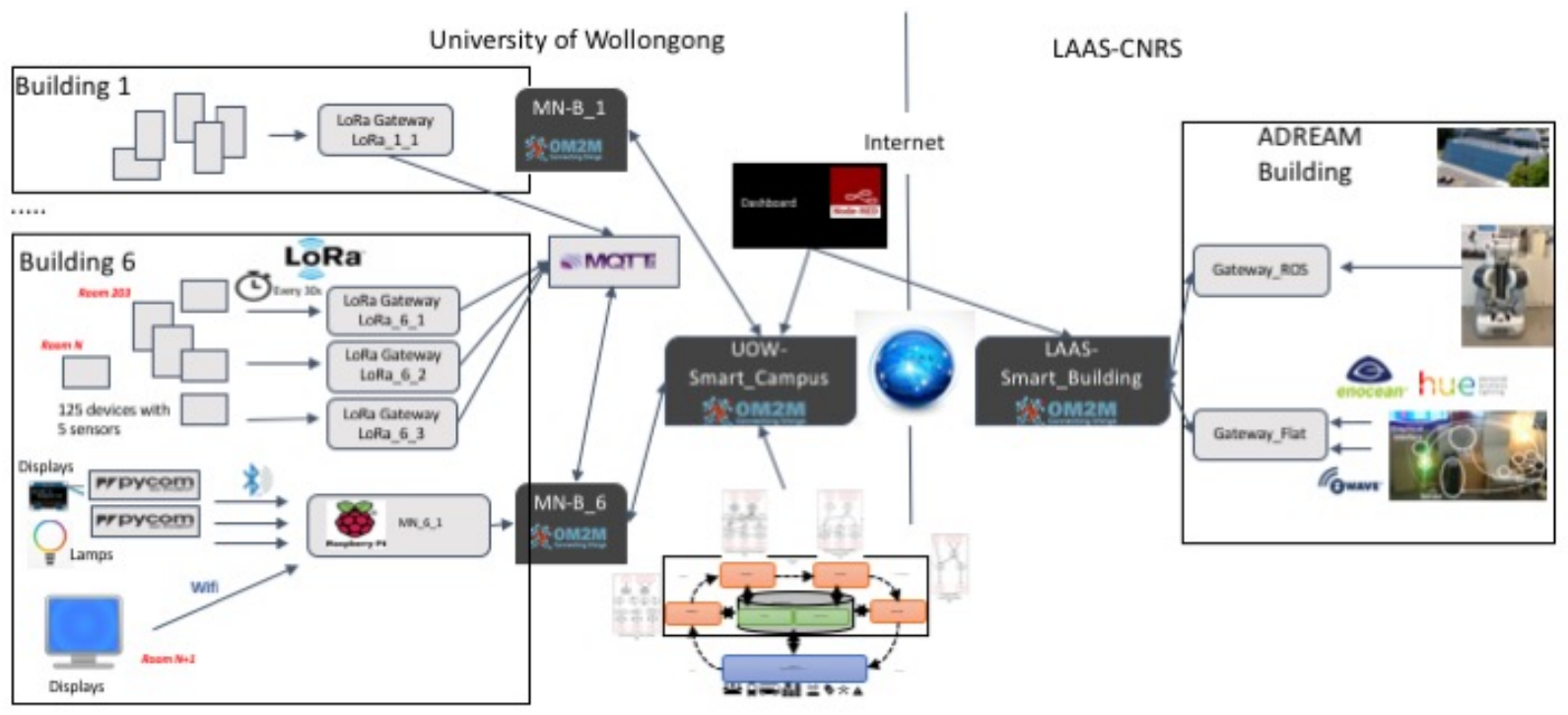

Fig. 6. A schematic view of the architecture showing both sites in France and Australia

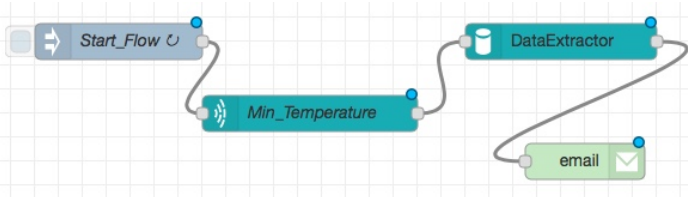

Fig. 7. A Node-RED flow to extract the minimum temperature

access to the teacher resource is provided to the student allowing them to consume questions and publish answers. A virtual resource modelling the student is also created with a container mark. The teacher is granted with privilege over this resource allowing him to send the results of a quiz session. The student application then subscribes to both the mark and the questions containers allowing automatic display of new questions and results.

The connection between the teacher and the students is made transparent by the usage of oneM2M and the autonomic composition of services by the ontology according to the interaction interest and the interaction context. Security is both guaranteed by a dynamic management of the Access Control Policies and the validation of the composition by the context matching.

\section{RELATED WORK}

The work introduced in this paper may be related to three themes: service composition, localization in the IoT and Context-Awareness. In this section, we position the contribution in regards to those three domains.

\section{A. Service composition}

Much work have been done to provide an automatic composition plan. The first aspect of composing services is providing composition methods and algorithms to determine which services to uses in order to fulfill a pre-established workflow.
For instance, in [16], [21] and [17] several contributions are studied. Different methods and algorithms are proposed based on AI algorithms, planning techniques or particular calculations which aim to provide an instantiated orchestration plan. Other studies focus more on enriching service meta-data with semantics to help the process of composing services such in [18] [19] and [20] where ontologies are proposed to enrich web services or REST services. Degas et al. [14] proposed an opportunistic composition of human-computer interfaces in ambient spaces using a cooperative multi-agent system as the core of the composition. A second aspect concerns the selection of the best service for the different parts of the workflow depending on QoS [22] or robustness of choice [23]. In this paper, we do not use any predefined workflow as the actions are determined on the fly, and the selection process is only driven by the context mechanisms.

\section{B. Localization in the IoT}

Many localization algorithms have been designed for wireless sensor networks for both indoor and outdoor environments [24]. For example, Alsinglawi et al. [15] have identified Radio Frequency Identification (RFID) technologies as a key enabler technology for the IoT due to its cost-effectiveness, high readability rates, automatic identification and energy efficiency characteristics. However, one of the major limitations of RFID technologies is that it needs to equip the environment with a set of pre-identified markers and thus limits the openness of the system. Thus, indoor localisation is still remaining a challenge [25]. In this paper, we propose to use a context approach instead of a localisation approach, by using the connectivity information of each device to validate the composition of a service. By doing so, we offer a novel way the think the to localize IoT devices. 


\section{Context-awareness}

Context awareness has been identified as a key feature for the Internet of Things [11]. There is a huge spectrum of applications based on context awareness. Bansal et al. [26] has identified context-awareness as a key requirement for designing personalized services in smart homes. Urbieta et al. proposed an adaptive context-aware service composition for IoT-based smart cities [27]. [28] evaluated the performance of semantic Web technologies to facilitate context-awareness and interoperability. Goel et al. [29] used context-awareness for smart water management in order to dynamically acquire a range of water-specific information in an IoT environment. This paper contributes to the research to enable contextawareness in the Internet of Things using autonomous ontologies management. Its novelty come from the usage of devices connectivity as a context to infer location and its application to two smart campuses.

\section{CONCLUSION}

This paper introduces a service oriented autonomic management approach for IoT systems with a specific application to smart campus. This framework relies on an ontology based model and context-awareness for composing new services on-the-fly. It is supported by the usage of the oneM2M middleware that provides interoperability and discoverability of data.

The proposed architecture enables IoT systems to cope with the dynamic and versatile nature of these systems and their environments by enforcing autonomic management actions while guaranteeing provisioning OF complex new value added services.

The architecture is being deployed on two different sites, at LAAS-CNRS and at the University of Wollongong, allowing replicability of the experiments and remote information exchange between the two sites.

Perspectives imply the deployment of more complex scenarios and experimental validation of the architecture performance.

\section{ACKNOWLEDGMENT}

In France, this work has been co-funded by the French Government (program: investment for future) in the project: Smart Services for Connected vehiCles - S2C2 and by INSA Toulouse with the program international mobility.

\section{REFERENCES}

[1] Overview of the Internet of things, ITU-T Y.4000/Y.2060, june 2102

[2] G. Garzone, N. Guermouche and T. Monteil, Autonomic Management Approach for Dynamic Service Based IoT Systems, IEEE International Symposium on Networks, Computers and Communications - ISNCC 2018, Roma, Italy, June 2018.

[3] N. Seydoux, K. Drira, N. Hernandez, T. Monteil, IoT-O, a Core-Domain IoT Ontology to Represent Connected Devices Networks. International Conference on Knowledge Engineering and Knowledge Management EKAW2016, Bologna, Italy, November, 2016.

[4] J.O. Kephart and D.M. Chess, The vision of autonomic computing, Computer, pp 41-50, Vol 36, 2003, doi 10.1109/MC.2003.1160055.
[5] T. Horrocks, P.F. Patel-Scneider, H. Boley, S. Tabet, B. Grosof and M Dean, SWRL: A Semantic Web Rule Language Combining OWL and RuleML, W3C Member submission 21, pp 1-20, may 2004

[6] Guivarch, V., Camps, V., \& Pninou, A. AMADEUS: an adaptive multiagent system to learn a users recurring actions in ambient systems. ADCAIJ: Advances in Distributed Computing and Artificial Intelligence Journal, 1(3), 1-10.2013

[7] Brezillon, P. Context in problem solving: a survey. The Knowledge Engineering Review, 14(1), 47-80. 1999

[8] Schilit, B. N., \& Theimer, M. M. Disseminating active map information to mobile hosts. IEEE network, 8(5), 22-32. 1994

[9] Abowd, G. D., Dey, A. K., Brown, P. J., Davies, N., Smith, M., \& Steggles, P. Towards a better understanding of context and contextawareness. In International symposium on handheld and ubiquitous computing (pp. 304-307). Springer, Berlin, Heidelberg. 1999

[10] Bazire, M., \& Brezillon, P.. Understanding context before using it. In International and Interdisciplinary Conference on Modeling and Using Context (pp. 29-40). Springer, Berlin, Heidelberg.2005

[11] Perera, C., Zaslavsky, A., Christen, P., \& Georgakopoulos, D. Context aware computing for the internet of things: A survey. IEEE communications surveys \& tutorials, 16(1), 414-454. 2014

[12] Verstaevel, N., Boes, J., \& Gleizes, M. P. From Smart Campus to Smart Cities: Issues of the Smart Revolution. 2017 IEEE SmartWorld, Ubiquitous Intelligence \& Computing, Advanced \& Trusted Computed, Scalable Computing \& Communications, Cloud \& Big Data Computing, Internet of People and Smart City Innovation (SmartWorld/SCALCOM/UIC/ATC/CBDCom/IOP/SCI), San Francisco, CA, 2017, pp. 1-6. 2017

[13] P. Piche, E. Belghache, N. Verstaevel and B. Lartigue, "Impact of ecofeedback on the behavior of campus users," 2017 IEEE SmartWorld, Ubiquitous Intelligence \& Computing, Advanced \& Trusted Computed, Scalable Computing \& Communications, Cloud \& Big Data Computing, Internet of People and Smart City Innovation (SmartWorld/SCALCOM/UIC/ATC/CBDCom/IOP/SCI), San Francisco, CA, 2017, pp. 1-6. 2017

[14] Degas, Augustin, et al. "Opportunistic Composition of Human-Computer Interactions in Ambient Spaces." Ubiquitous Intelligence \& Computing, Advanced and Trusted Computing, Scalable Computing and Communications, Cloud and Big Data Computing, Internet of People, and Smart World Congress (UIC/ATC/ScalCom/CBDCom/IoP/SmartWorld), 2016 Intl IEEE Conferences. IEEE, 2016.

[15] Alsinglawi, B., Elkhodr, M., Nguyen, Q. V., Gunawardana, U., Maeder, A., \& Simoff, S. J. RFID localisation for Internet of Things smart homes : a survey. International Journal of Computer Networks and Communications, 9(1), 81-99. 2017.

[16] K. S. M. Chan, J. Bishop, and L. Baresi, Survey and comparison of planning techniques for web services composition, Africa (Lond)., no. October, pp. 4354, 2007.

[17] D. Hutchison and J. C. Mitchell, Semantic Web Services and Web Process Composition, vol. 3387. Berlin, Heidelberg: Springer Berlin Heidelberg, 2005.

[18] R. Dumitru, K. Uwe, L. Holger, B. de Jos, L. Rubn, S. Michael, P. Axel, F. Cristina, B. Cristoph, and F. Dieter, Web Service Modeling Ontology, Appl. Ontol., vol. 1, no. 1, pp. 77106, 2005.

[19] T. Vitvar, J. Kopeck, J. Viskova, and D. Fensel, WSMO-lite annotations for web services, Lect. Notes Comput. Sci. (including Subser. Lect. Notes Artif. Intell. Lect. Notes Bioinformatics), vol. 5021 LNCS, pp. 674689, 2008.

[20] D. Roman, J. Kopeck, T. Vitvar, J. Domingue, and D. Fensel, WSMOLite and hRESTS: Lightweight semantic annotations for Web services and RESTful APIs, J. Web Semant., vol. 31, pp. 3958, 2015.

[21] M. Aljawarneh, L. Dhomeja and Y.A. Malkani, Yasir Arfat, Contextaware Service Composition of Heterogeneous Services in Pervasive Computing Environments : A Review, 19th International Multi-Topic Conference (INMIC), Islamabad, Pakistan, 2016.

[22] R. J. R. Raj, T. Sasipraba, Web service selection based on QoS Constraints, Trendz in Information Sciences Computing (TISC), Vol 6, pp 156-162, doi 10.1109/tisc.2010.5714629, 2010.

[23] I. Guidara, T. Chaari, K. Fakhfakh, Intention based semantic approach for service sourcing, 27th Annual $\{\mathrm{ACM}\}$ Symposium on Applied Computing, New York, 2012.

[24] Chen, Zhikui, et al. "A localization method for the Internet of Things." The Journal of Supercomputing 63.3 : 657-674. 2013 
[25] McConville, Ryan, et al. "Understanding the quality of calibrations for indoor localisation.” Internet of Things (WF-IoT), 2018 IEEE 4th World Forum on. IEEE, 2018

[26] Bansal, Maggi, Inderveer Chana, and Siobhan Clarke. "Enablement of IoT based context-aware smart home with fog computing." Fog Computing: Breakthroughs in Research and Practice. IGI Global, 251263. 2018

[27] Urbieta, Aitor, et al. "Adaptive and context-aware service composition for IoT-based smart cities." Future Generation Computer Systems 76 (2017): 262-274.

[28] Maarala, Altti Ilari, Xiang Su, and Jukka Riekki. "Semantic reasoning for context-aware Internet of Things applications." IEEE Internet of Things Journal 4.2 (2017): 461-473.

[29] Goel, Deepti, Santanu Chaudhury, and Hiranmay Ghosh. "Smart Water Management: An Ontology-Driven Context-Aware IoT Application." International Conference on Pattern Recognition and Machine Intelligence. Springer, Cham, 2017. 